The counting of the Chart plates has been continued during the year, and completed between Dec. $64^{\circ}$ and Dec. $70^{\circ}$. A paper on the statistics of the stars between $65^{\circ}$ and $70^{\circ}$ N. Dec. was communicated to the Royal Astronomical Society in January, and printed in the Monthly Notices.

The 28 -inch refractor has been used throughout the year for micrometric measurements of double stars. The total number of double stars measured during the year is $38 \mathrm{r}$; of these 192 have components less than $I^{\prime \prime} \cdot 0$ apart, and xo5 less than $0^{\prime \prime} \cdot 5$.

Series of measures have been obtained of $\kappa$ Pegasi, $\delta$ Equulei, 7o Ophiuchi, and $\delta$ Herculis. Capella has been examined at every favourable opportunity, and observations of the position angle of the elongated image have been secured on eight occasions.

Solar Activity.- Shortly after the date of the last report a long period of almost complete solar quiescence set in: from 1902 June 5 to September 17 inclusive, a period of I05 days, the mean daily spotted area was less than a single unit (one millionth of the sun's visible hemisphere). An active period set in on September is and lasted until November 28,72 days, the mean daily area being 164 millionths. The rest of the year 1902 was very quiet, the remaining 34 days showing a mean daily area of only 3 . In the present year the sun has been much more active, and has been free from spots on only I4 days since January I, as compared with about 100 in the same period of last year. The first of a fine series of spot-groups appeared on the east limb on 1903 March 21 , and a succession of new groups has followed almost without intermission up to the date of this report. There can be no doubt, therefore, that the solar activity is very decidedly upon the increase.

Tables and diagrams showing the distribution of sunspots in latitude and the areas of sun-spots and faculæ compared with magnetic diurnal ranges for the 29 years 1874 to 1902 have been prepared, and will be published in the Monthly . Votices R.A.S. for May.

Magnetic Observations. - The principal results for the anagnetic elements for 1902 are as follows :-

Mean declination $16^{\circ} 22^{\prime} \cdot 8$ West

Mean horizontal force ... ... $\left\{4^{\circ} \mathrm{Or} 34\right.$ (in British units).

Mean dip (with 3 inctsic units).

3 -inch needles) $67^{\circ} 3^{\prime} 25^{\prime \prime}$

Meteorological Observations. - The mean temperature for the year 1902 was $49^{\circ} \cdot \mathrm{I}$, or $0^{\circ} \cdot 4$ below the average for the 50 years $184 \mathrm{I}-90$.

The rainfall for the year ending I903 April 30 was 23.68 inches, being 0.86 inch less than the average of 50 years. The number of rainy days was 172 . The rainfall has been less than the average for each of the eight years from 1895 to 1902 inclusive, the total deficiency for the eight years ending 1902 December 31 amounting to 28.91 inches. For the four months igo3 January-April there has been an excess of 0.95 inch.

\section{THEORY OF CYCLONES AND ANTICYCLONES}

PROF. F. H. BIGELOW contributes to the U.S.

Monthly Weather Review for February a paper on the mechanism of counter-currents of different temperatures in cyclones and anticyclones. An outline theory of the structure of cyclones and anticyclones was described in the repcrt of the Chief of the Weather Bureau for 1898 1899 (vol. ii). It was evident, however, that a more complete insight into the mechanism of motions in a fluid such as air under atmospheric conditions would be afforded by the construction of systems of isobars on at least three planes having different altitudes. For this purpose, the sea-level and the levels of 3500 and 10,000 feet were selected, and since December, I902, daily reduced pressures for these planes have been received from the regular observing stations of the United States and Canada, and charts have been constructed for them. The approximate gradients needed for a preliminary consideration of the subject have thus been obtained, and the general results of the investigation are stated by Prof. Bigelow as follows :-

(I) The cyclone is not formed from the energy of the latent heat of condensation, however much this may strengthen its intensity; it is not an eddy in the eastward drift; but it is caused by the counterflow and overflow of currents of different temperatures. Ferrel's canal theory of the general circulation is not sustained by the observations, nor is his theory of local cyclones and anticyclones tenable. There are difficulties with regard to the German vortex theory, but this is nearer the truth than the Ferrel vortex. The structure in nature is actually more complex than has been admitted in these theoretical discussions, but it doubtless can be worked out successfully along the lines herein indicated. (2) Regarding the relation of the upper level isobars to practical forecasting, it is noted as the result of the examination of charts that $(a)$ the direction of the advance of the centre of the low pressure is controlled by the upper strata, and its track for the following twentyfour hours is usually indicated by the position of the ro, 000foot level isobars; (b) the velocity of the daily motion is also dependent upon and is shown by the density of these high level isobars; $(c)$ the penetrating power of the cyclone is safely inferred from an inspection of the three maps of isobars of the same date; $(d)$ there is decided evidence that areas of precipitation occur where the 3500 -foot isobars and the $10,000-f o o t$ isobars cross each other at an angle in the neighbourhood of $90^{\circ} ;(e)$ there have been several cases in which the formation of a new cyclone has been first distinctly shown on the upper system of isobars before penetrating to the surface or making itself evident at the sea level. (3) It is expected that by completing our discussion of the temperature gradients between the surface and the higher levels we shall be able to secure daily isotherms as well as daily isobars on the upper planes, and this will tend to strengthen any further examination of these important problems. A suitable report will be prepared in which the data now coming into our possession will be subjected to a mathematical analysis and discussion.

\section{ATMOSPHERIC VARIATIONS.}

F ROM the results of recent researches solar prominences seem to be playing a most important part, not only in the mechanism of the solar atmosphere, but in the variations of our own. Any investigation, therefore, that gives us new ideas or corroborates the old is most useful and valuable. In a previous number of this Journal (vol. lxvii. p. 569, April) an account was given of the results obtained from a research on the distribution of solar prominences as regards latitude. The prominence circulation thus disclosed that there was practically a law at work which the centres of prominence action followed, and this law, deduced from observations extending over the longest period available (1872-1901), was found to be in good agreement with that first suggested by Prof. Ricco in 1891 (Mem. d. Soc. degli Spettr., vol. xx. p. r35). Prof. Bigelow has also been studying the question of prominence, facula and spot circulation, and in a recent number of the Monthly Weather Review (vol. xxxi. No. I, p. 9) has stated his results. The method he adopted was somewhat different from the one first mentioned above, for the prominence circulation determined by him has been deduced by finding the mean variation of the prominence distribution resulting from coupling up together the values for those years which he considers are similar in relation to the eleven-year sun-spot cycle. Anyone familiar with this cycle knows the difficulty this involves, because it is only the mean length of the sun-spot period that is eleven years. Further, the epochs of maxima do not follow those of the minima at constant intervals, but vary from a little more than three to five years. In spite, however, of these probable sources of error, Prof. Bigelow deduces a circulation not very different from the one mentioned above, so that all the three computations and deductions show that there is a very definite movement in latitude and change in percentage frequency of occurrence from year to year.

$A$ most interesting and important contribution, by Prof. T. H. Davis, to our knowledge of the fluctuation of the annual wind resultants, and indirectly to our knowledge of the movements of cyclones and anticyclones, appeared in one of the recent numbers of the Monthly Weather Review (vol. xxx. No. II, p. 5ig). The investigation was restricted chiefly to stations included in the meteorological services of the United States and Canada, and the period discussed was the ten years isgi-igoo. The results of the research 